\title{
New Perspectives from Misdiagnosis: A Case of Primary Cutaneous Cryptococcosis Treated with Hydroxychloroquine Sulfate Successfully
}

Received: 26 March 2020/Accepted: 5 May 2020/Published online: 17 May 2020

(C) Springer Nature B.V. 2020

A 20-year-old man from Southwest China was admitted to the outpatient Department of Dermatology with a 3-month history of proliferative plaque on his nose. The lesions began as pruritic papules that progressively ulcerated. Physical examination found one well-demarcated proliferative plaques with pustules and overlying yellow-brown crust (Fig. 1A). Before the eruption, no trauma or other clear source of infection was identified except for chronic scratching. The patient was otherwise healthy with no systemic symptoms or history of immunosuppression. All other serum chemistry values were within normal limits. The HIV antibody test was negative. Secretion bacterial culture was negative. The same white yeast-like colonies were obtained after 3 days with multi-point inoculation of skin tissue and secretions on SDA at $28{ }^{\circ} \mathrm{C}$, and the fungus was identified empirically as Prototheca species due to being unicellular measuring $>10 \mu \mathrm{m}$ with endospores and without capsule. A skin biopsy was performed, and Gomori methenamine silver stains showed numerous round yeast cells without capsule invading skin and subcutaneous tissue, more easily visible within giant cells. Based on clinical and morphological characteristics of pathogens (Fig. 2), cutaneous protothecosis was initially diagnosed and systemic therapy with oral itraconazole $400 \mathrm{mg} /$ day was started, and Rash relief was not obvious after 1 month of treatment. Taking into account that Prototheca species in general have low susceptibility to antimicrobial agents, and the lesions occurred on exposed areas similar as lupus, hydroxychloroquine sulfate $400 \mathrm{mg}$ daily was first attempted to treat this rare infection, minocycline $100 \mathrm{mg} /$ day was combined at the first 2 months, and the reduction of itraconazole was $200 \mathrm{mg}$ per day for 2 months. Strikingly, the lesion improved after 1 month and completely resolved after 6 months (Fig. 1B). The lesions did not recur and no side effects appeared during 1 year of follow-up. Based on molecular sequencing, the fungus was identified as Cryptococcus neoformans var. grubii, and the final diagnosis was corrected as primary cutaneous cryptococcosis due to no clinical evidence of disseminated disease. The ITS nucleotide sequences were deposited in GenBank with accession number MT421896. We did misdiagnose the patient as cutaneous protothecosis initially due to our empirical morphological knowledge, but this special experience gave us the chance to re-examine the old drug, except for malaria parasite and spirochaete infections, and more potential antimicrobial activity of hydroxychloroquine sulfate should be further explored, such as antifungal or coronavirus activity. 

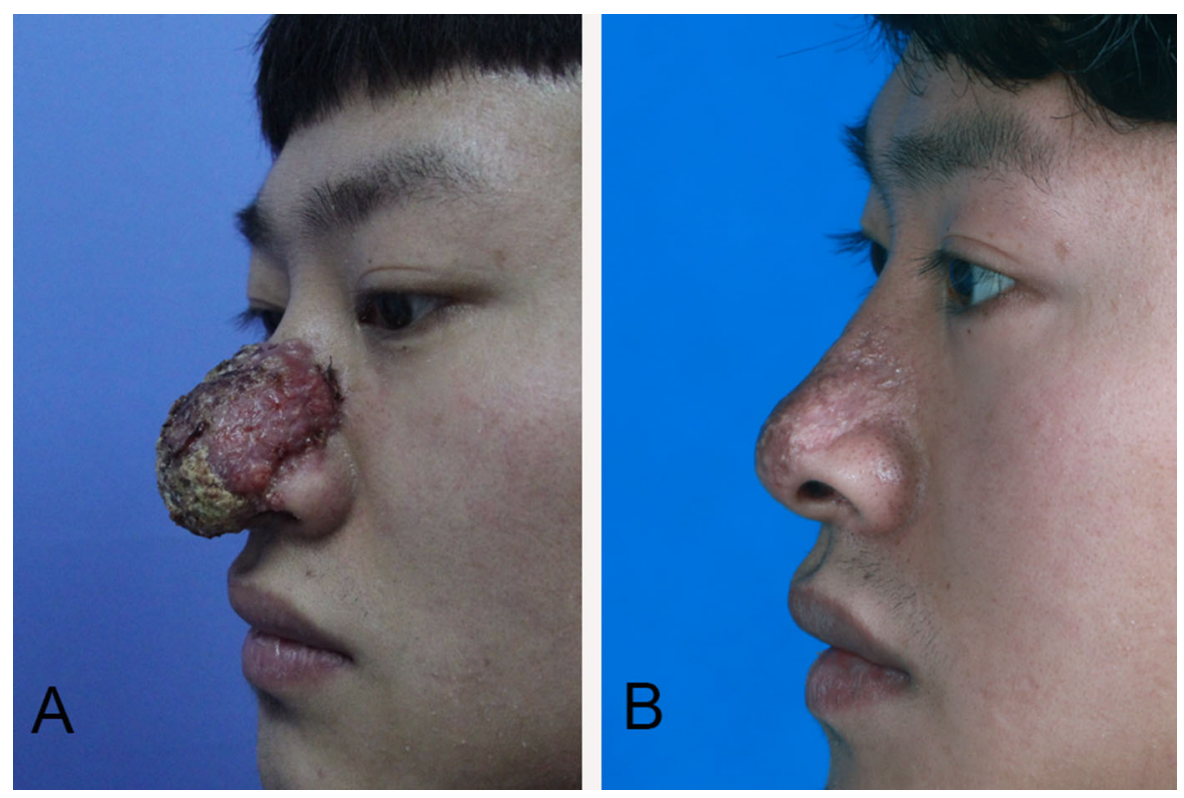

Fig. 1 One well-demarcated proliferative plaques with pustules and overlying yellow-brown crust (A); resolution of lesions with atrophic superficial scar after 6-month treatment $(\mathbf{B})$

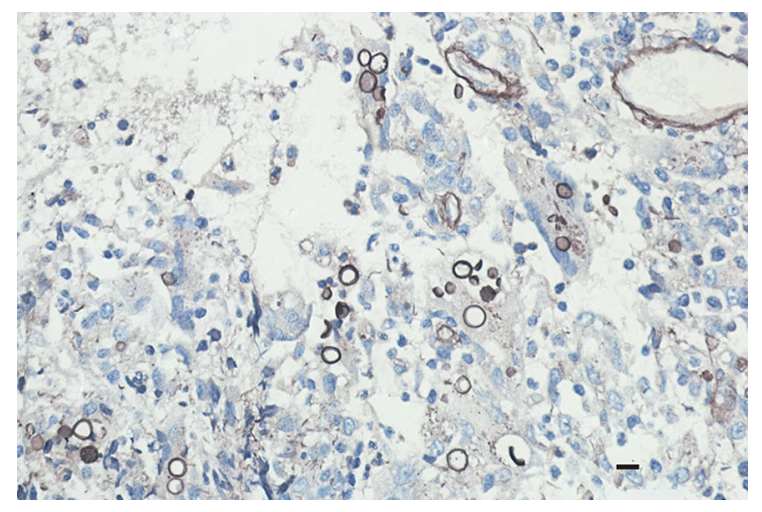

Fig. 2 Gomori methenamine silver stains showing numerous round yeast cells without capsule invading skin and subcutaneous tissue, more easily visible within giant cells. Scale bar $10 \mu \mathrm{m}$

\section{Compliance with Ethical Standards}

Conflict of interest The authors declare no conflict of interest.
Ethical Statement The authors confirm that this material is original and has not been published in whole or in part elsewhere; that the manuscript is not currently being considered for publication in another journal; and that all authors have been personally and actively involved in substantive work leading to the manuscript, and will hold themselves jointly and individually responsible for its content.

Publisher's Note Springer Nature remains neutral with regard to jurisdictional claims in published maps and institutional affiliations.
${ }^{1}$ Wanglan Luo
${ }^{1}$ Di Che
${ }^{1}$ Hongguang Lu
${ }^{1,2}$ Yanping Jiang (D)

${ }^{1}$ Department of Dermatology, The Affiliated Hospital, Guizhou Medical University, Beijing Road 4, Yunyan District, Guiyang, China e-mail: jiangyanping119@163.com

${ }^{2}$ Centre of Expertise in Mycology of RadboudUMC/CWZ, Nijmegen, The Netherlands 